ing destruction and has become inadequate for the continuing evolution of man. We must develop a new species to succeed Homo sapiens sapiens." Skinner's stratagem to apply behavioural science to avert the end of man is considered to be futile ("How can a controlled society be designed by behaviourists in which unknown change of man is permitted and encouraged?"). there are genuflexions to Teilhard de Chardin and overtures to ESP. Yet Ts'o never asks how we can hope to determine exactly which genetic changes are needed in order to alter human behaviour predictably.

The other chapters are invaluable advanced reviews by authors who have each made leading contributions to their topics. Some, such as Eisenberg and Bauer and Vinograd, have succeeded in being critical and selective, but the general tone is encyclopaedic. Thus, the volumes are more likely to be used as a treasure house of reference data than as teaching material, except in very specialised postgraduate work. In this respect the title of the series is misleading. Volume 1 has a comprehensive chapter on chemical synthesis and transformations of nucleosides (L. Goodman), followed by clear, stimulating accounts of mass spectrometry (McCloskey) and excited states (Gueron, Eisinger and Lamola). Tsuboi's chapter on infrared and Raman spectroscopy assumes a prior knowledge of Raman spectroscopy and the To spice the mixture of arguments,

volume ends with a long chapter by the editor on the structure of bases, nucleosides and nucleotides and their interactions with each other and with polymers.

Volume 2 is of more general interest to biochemists. It has excellent chapters on the chemical reactions of polynucleotides and nucleic acids (D. M. Brown), ultraviolet spectroscopy, circular dichroism and optical rotatory dispersion (Bush), hydrodynamic and thermodynamic studies (Eisenberg) and on circular DNA (Bauer and Vinograd). A final chapter by Ts'o is on the conformations and interactions of dinucleotides and oligonucleotides. The two chapters by Ts'o at the end of each volume are long and very detailed indeed, but they are in a field in which he has made important and extensive contributions. Naturally, there is some overlap with other chapters but it is often useful to discuss the material in a different context.

Although the preface remarks on the one hundred years since Miescher, it is more to the point that nineteen years have passed since the publication of the first two volumes of The Nucleic Acids edited by Chargaff and Davidson. Despite the presence of irrelevant material, the present volumes are particularly welcome, since the physicochemical aspects of nucleic acids have been reviewed far less than their biology and enzymology. We can look forward to the later volumes of the series.

K. Burton

\section{All about the gibbon and the siamang}

Gibbon and Siamang. Vol. 3: Natural History, Social Behaviour, Reproduction, Vocalization, Prehension. Edit. by Duane M. Rumbaugh. Pp. vii +208 . (Karger: Basel and London, 1974.) SFr.130; £18.85; \$40.30; DM124.

THIS volume contains one long paper by Ellefson, reporting a field study on the white-handed gibbon Hylobates lar, and three much shorter papers. These are, respectively, an account by Brockelman, Ross and Pantuwatana of the social behaviour of a small experimental colony of $H$. lar on an island in the Gulf of Thailand, a description in terms of functional morphology of the hylobatid hand by $\mathrm{R}$. Lorenz and an analysis of vocalisations from captive gibbons and siamangs by Tembrock.

Unfortunately, much of the value arising from the fact that the papers are published together in the one book, rather than appearing separately in journals, is lost by the fact that there is no liaison between them. For example, when Brockelman et al. and Lorenz refer to Ellefson's work they refer to his unpublished doctoral dissertation rather than to his paper in this book, and Tembrock's paper contains no reference to Ellefson's work at all. This lack of liaison may have been no fault of the authors, but it does point to poor planning in the production of the book.

The three short papers are each useful additions to the primate literature. Ellefson's paper is more of a problem. The blurb on the dust jacket says "Observational data have been reduced when appropriate to quantitative summaries, but their interpretation and/or possible meaning is never lost." This policy seems little short of disastrous. It ensures that no reader can check the validity of Ellefson's conclusions against his data, nor does it allow detailed quantitative comparisons with published data from other primate field studies. In addition to the cavalier treatment of data, the treatment of theoretical issues, such as the aetiology of vocalisations and the concept of dominance, are naive in comparison with the relatively sophisticated literature that has been published on these topics in recent years. It is disappointing that what was clearly such a diligent study should have been so poorly $\begin{array}{ll}\text { reported. } & \text { N. R. Chalmers }\end{array}$

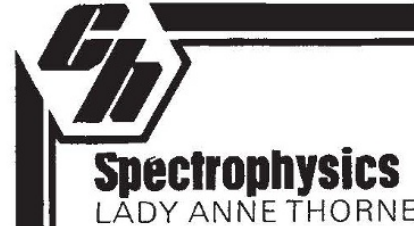

LADY ANNE THORNE

Hardback: September 1974 : 416 pages: 163 line illustrations: $412125102: £ 6.50$ Science Paperback : 41212530 $X: £ 3.90$

Spectrophysics describes the main methods of experimental spectroscopy and their application to the study of physica! phenomena, with particular reference to the determination of transition probabilities and to problems in laboratory and astrophysical plasmas.

\section{SEVENTH EDITION}

\section{Insect Physiology}

SIRV. B.WIGGLESWORTH.

\section{F.R.S.}

Hardback: October 1974: 176 pages: 20 line illustrations: $412111500: £ 2.95$

Science Paperback : 41220980 $2: £ 1.65$

The seventh edition of this readable introductory account of the physiology of insects has been completely updated and includes a new chapter on insect hormones. This book will be intelligible to any reader with no more than an elementary knowledge of biology but at the same time it gives a very complete survey of the subject. References are given to more complete treatment where necessary.

MATHEMATICS SERIES

\section{Iniroduction to Optimization Methods}

P. R. ADBY and

M. A. H. DEMPSTER

Limp Binding: October 1974 : 214 pages: 41211040 7: $£ 2.50$

This book is an introduction to non-linear methods of optimization. The basic methods of single and multi-parameter optimization, presented here in a simple uniform terminology. are an essential prerequisite to a study of the current advanced methods reviewed under a few generic headings in the final two chapters.

Further information on these titles and a list of stockists is available from the publishers on request.

CHAPMAN \& HALL 11 NEW FETTER LANE, LONDON EC4P 4EE 